\title{
HOT MELT EXTRUSION IN ENGINEERING OF DRUG COCRYSTALS: A REVIEW
}

\author{
ARCHANA RAJADHYAX, UJWALA SHINDE, HARITA DESAI*, SHRUSHTI MANE
}

Department of Pharmaceutics, Bombay College of Pharmacy, Mathuradas Colony, Mumbai, Maharashtra, India. Email: harita.desai@bcp.edu.in

Received: 20 April 2021, Revised and Accepted: 08 June 2021

ABSTRACT

Crystal engineering technique has been widely explored in recent times to bring about changes in crystallinity which aids to achieve various goals such as solubility enhancement, stability and in vivo bioavailability without altering the chemical properties of the drug. Cocrystallisation is one of the crystal engineering approaches where the drug and an inert coformer are linked together by hydrogen bonding forming supramolecular homosynthon or heterosynthon using solvent-based or solvent-free techniques. Processing of active pharmaceutical ingredients with inert watersoluble coformers yields multicomponent crystalline cocrystals with high-performance characteristics and enhanced flow properties. Due to the emerging need of the industry for greener techniques, hot melt extrusion (HME), a continuous and solvent-free process is emerging as a field of interest in the mechanochemical synthesis of various pharmaceutical dosage forms such as solid dispersions, implants, ointments, and cocrystals. The current review emphasizes the role of HME as a cocrystallization technique for drugs to tailor-make their properties and ease of formulation. The distinct feature of HME is phase control during the process of cocrystallization. Furthermore, the selection of appropriate coformers with desirable water-solubility and stability features makes HME amenable to cocrystallization of versatile actives yielding suitable dosage forms. The application of process analytical technology further adds ease of monitoring during HME in cocrystallization approaches. Due to these salient features of HME, it can act as a prospective technique for cocrystallization of versatile drugs thus yielding dosage forms with desirable solubility and stability features.

Keywords: Crystal engineering, Cocrystallization, Solvent-free, Hot melt extrusion, Screw extruders, Synthons.

(C) 2021 The Authors. Published by Innovare Academic Sciences Pvt Ltd. This is an open access article under the CC BY license (http://creativecommons.org/ licenses/by/4.0/) DOI: http://dx.doi.org/10.22159/ajpcr.2021v14i8.41857. Journal homepage: https://innovareacademics.in/journals/index.php/ajpcr

\section{INTRODUCTION}

Combinatorial chemistry and high-throughput screening have paved a way for new drug candidates by which innumerable new molecules are generated. However, most of them are unable to be transformed as final dosage form due to accompanied issues of solubility, stability, and bioavailability. This gives rise to a need for tailor-making these molecules to make them amenable for preparing formulations, exhibiting desired therapeutic action.

Various formulation strategies are designed based on the biopharmaceutical properties of drugs. The most useful tool for decision-making is the biopharmaceutics classification system (BCS) which categorizes drug substances in four categories depending on their solubility and intestinal permeability as shown in Fig. 1. BCS has been utilized by regulatory agencies to establish the in vivo bioequivalence utilizing in vitro dissolution profiles of drug substances. BCS plays a major role in developing biowaivers as well as for formulation design from early to clinical stages $[1,2]$

Particle engineering is a viable option to modify physicochemical, particulate and biopharmaceutical properties of the poorly soluble drugs to improve their solubility. Particle engineering comprises of physical modifications, crystal and chemical modifications. Physical modifications include size reduction yielding microparticles, nanoparticles and nanosuspensions. Crystal modifications yield amorphized drugs in solid dispersions. Chemical modifications involve complexation (with cyclodextrins), $\mathrm{pH}$ modification, derivatization, salt formation and cocrystal formation [3]. Miscellaneous solubilization techniques explored for solubility enhancement include the addition of surfactant, emulsification, bilayered systems (liposomes), solid lipid nanoparticles, polymeric micelles, nanostructured lipid carriers, supercritical fluid method, solubilizers, cosolvency, and hydrotrophy [4]. Solubilization approaches such as the use of cosolvents, micelle formation and complexation are mainly used for decreasing lipophilicity [5]. Similarly for altering the intermolecular forces, solid-state modifications can be utilized such as formation of polymorphs, cocrystals or solvates [6].

SEDDS can cause carriers to strongly adsorb and physically interact with the drug resulting in retarded or incomplete drug release [3]. The addition of surfactants may cause GIT irritation. Salt formation approach is not feasible for weakly ionizable or neutral molecules. The $\mathrm{pH}$ modification approach is not suitable for drugs exhibiting pH-dependent stability. Size reduction techniques aid to enhance dissolution rate without affecting equilibrium solubility. Micronization shows enhanced Van der Waals interaction and electrostatic attraction reducing the effective surface area for dissolution impacting bioavailability adversely. Controlling particle size becomes difficult leading to heterogeneous particle shapes [7]. Metastable polymorphs and stabilized high energy amorphous systems such as solid dispersions show solubility enhancement however they are not effective in achieving adequate physicochemical stability and appropriate processing properties [8]. Hence there arises a need to evaluate and develop innovative technologies for producing dosage forms of drugs with varying solubility profiles.

\section{CRYSTAL ENGINEERING: SALIENT FEATURES}

Crystal engineering involves the design of solid-state structures using noncovalent interactions between ionic or molecular components yielding endproducts with altered physicochemical attributes amenable for dosage form designing [9]. Besides solid-state interaction, control, rationalization and structure prediction, the study can be utilized to tailor-make drug properties [10]. Crystal engineering technique can be applied to poorly-soluble drug molecules with versatile therapeutic profiles to formulate bioavailable dosage forms. Furthermore, the technique can also be applied to water soluble drugs with stability issues to enhance the stability of the active thus aiding in producing dosage forms stable throughout the shelf life. Changes at the intrinsic crystalline level are possible using crystal engineering approach. 
Crystallization occurs in the solution or melt of the crystalline compound. Key processing parameters include the formation of crystal nuclei, influence of crystallization conditions, concept of growth unit and how the overall crystal structure evolves. The term "synthon" describes synthetic organic structural features. A "supramolecular synthon" forms the structural and functional unit of cocrystal within supermolecules which is designed by planned structured noncovalent type of intermolecular interactions. Crystal engineering focuses on designing robust preconceived synthons utilizing intermolecular interactions that yield multicomponent crystalline entities with features suitable for formulation development. The Cambridge structural database (CSD) is used for identifying stable hydrogen bonding motifs ensuring that the most robust motifs remain intact across a family of related structures [11]. The supramolecular synthons are classified as Homosynthon when the hydrogen bond is formed between the same functional groups possessed by the API and coformer, for example, acid dimer, amide dimer or heterosynthon when two different functional groups possessed by the API and coformer are involved in forming a hydrogen bond. For example, amide-acid heterosynthon and acidpyridine heterosynthon $[12,13]$.

The crystallization process comprises of formation of nuclei which subsequently undergo crystal growth to form the specific 3D arrangements known as crystal lattice. Processing of APIs is mainly based on solution-based crystallization where solubility plays an important role. Crystallization occurs from the completely solubilized state due to the magnitude of difference in solubility that occurs at a specified solvent composition and temperature [14].

Habit modification in the crystallization of API leads to the exposure of different crystal faces which influences wettability and subsequent dissolution. There are various examples in the literature demonstrating the change in crystal habit and its influence on in vitro dissolution. For example, rod-shaped particles of dipyridamole crystallized from benzene showed a higher rate of dissolution than rectangular needleshaped crystals produced using methanol suggesting the effect of solvent on crystallization [15]. Studies conducted with phenytoin indicated the morphology of crystals recrystallized from ethanol to be needle-shaped while crystals from acetone showed rhombic morphology in spite of being produced under similar conditions. Thus, habit modification was attributed to stronger interaction of acetone with the hydroxyl groups of phenytoin due to its relatively high polarizability [16]

To produce powders with high purity and well-defined particle size distribution, controlled crystallization is the approach adopted. Melt sonocrystallization is an emerging technique where ultrasonic energy is utilized for the production of porous fast-dissolving particles for hydrophobic drug molecules $[17,18]$. Furthermore, various techniques

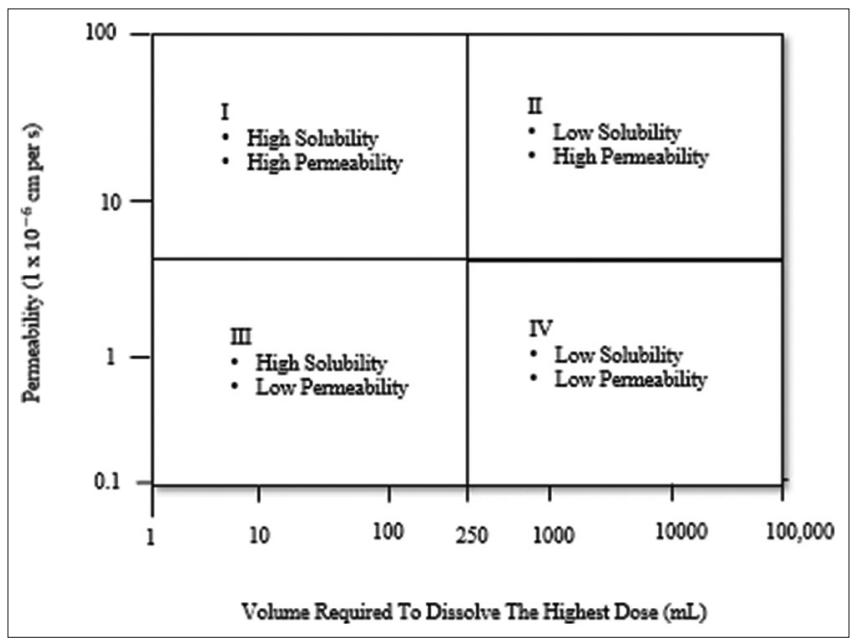

Fig. 1: Biopharmaceutics Classification System classification system Adapted from reference [2] based on supercritical fluid methodologies can be used for achieving submicron sizes for therapeutic agents [19].

\section{Cocrystallization as an innovative formulation approach}

To overcome the issue with the above-mentioned techniques and to produce a stable system, crystal engineering of cocrystals has developed a major interest. Cocrystals are solid crystalline single-phase materials consisting of two or more molecular compounds (API and Coformer) mixed in a fixed stoichiometric ratio. Coformer should possess hydrogen bonding functionalities to form supramolecular synthon with the API. The supramolecular library for cocrystallizing agents is been developed by referring to the CSD where they are classified according to their contribution to crystal packing arrangement depending on the functional groups of the parent molecule. As both the components here are solid, there is no stability issue involved. Furthermore, cocrystals can be crystallized into various polymorphic forms. Twenty such examples which include caffeine and glutaric acid polymorphic cocrystals have been reported to date. Coformers used are Generally Recognized as Safe listed. Cocrystallization between two APIs is also proposed where both the APIs can be employed in their sub therapeutic level to yield synergistic action [20].

Formation of cocrystals with different coformers can be predicted using the following approaches;

- Synthon engineering

- Phase diagrams

- pKa based

- Lattice energy calculations

- Hydrogen bonding propensity

- Hansen solubility parameters.

Cocrystals are advantageous as they exist in stable crystalline form without the need for any other excipients and additives in formulations. Furthermore, an increase in solubility and hence the dissolution rate an increases bioavailability. Processes such as micronization are not needed. The relative amount of solvent needed is also less thus making it a greener approach for obtaining dosage form with an increased solubility [21].

Various techniques are being used in cocrystallization such as solvent evaporation, grinding, solvent assisted grinding, antisolvent precipitation, ultrasonication, and hot melt extrusion (HME) $[22,23]$. Cocrystallization alters various physical properties of drugs such as melting point, solubility and enhances the chemical stability of the API. The above factors in turn aid in the enhancement of drug bioavailability. Marketed cocrystal products have been found to show moderate growth due to significant experimental efforts and regulatory risks related to approval (Table 1). Sildenafil citrate cocrystal marketed as Viagra (Pfizer) is used to treat male erectile dysfunction and pulmonary arterial hypertension.

Table 1: Marketed cocrystals [21-23]

\begin{tabular}{|c|c|c|c|}
\hline $\begin{array}{l}\text { Brand } \\
\text { name }\end{array}$ & $\begin{array}{l}\text { Drug used in } \\
\text { cocrystal }\end{array}$ & $\begin{array}{l}\text { Therapeutic } \\
\text { indication } \\
\text { of the drug } \\
\text { cocrystal }\end{array}$ & References \\
\hline Entresto $^{\circledR}$ & Sacubitril - Valsartan & Heart failure & {$[21,22]$} \\
\hline Lexapro ${ }^{\circledR}$ & Escitalopram oxalate & $\begin{array}{l}\text { Depression and } \\
\text { anxiety }\end{array}$ & [23] \\
\hline Depakote $^{\circledR}$ & $\begin{array}{l}\text { Valproate sodium - } \\
\text { Valproic acid }\end{array}$ & $\begin{array}{l}\text { Seizures, Maniac } \\
\text { depression }\end{array}$ & {$[22,23]$} \\
\hline Steglatro ${ }^{\circledR}$ & $\begin{array}{l}\text { Ertugliflozin } \\
\text { cocrystal - } \\
\text { 5-0xoproline }\end{array}$ & Antidiabetic & [21] \\
\hline Abilify ${ }^{\circledR}$ & $\begin{array}{l}\text { Aripiprazole - } \\
\text { Fumaric acid }\end{array}$ & Schizophrenia & [23] \\
\hline Suglat $^{\circledR}$ & $\begin{array}{l}\text { Ipragloflozin - } \\
\text { L-proline }\end{array}$ & $\begin{array}{l}\text { Antidiabetic } \\
\text { (Type-I) }\end{array}$ & [22] \\
\hline
\end{tabular}


From the current industrial perspective, the process must be continuous with fewer process variables, should be a green technique and also should have low production cost. All these make HME a technology of choice for cocrystallization.

\section{THE TECHNOLOGY OF HME}

HME is a process in which a drug is embedded in the melted polymeric matrix of thermoplastic polymers, low-melting waxes, sugar alcohols, starches or carrier systems with desirable features of solubility and stability. The processing aids to modulate different physicochemical attributes of the actives such as solubility, and stability. For successful processing, the physical state, melting point and molecular weight of drug and polymer exhibit an important role. The thermal stability of the individual components is very important. Thermolabile compounds can be processed at lower processing temperature and time; taking care to prevent degradation. During the processing comprising of polymers as carriers, plasticizers may be added which act as processing aids [24].

If the excipients used in HME process exhibit required pharmaceutical properties and safety parameters, the excipients can aid to yield final products with desirable bioavailability and stability characteristics. A variety of end products such as granules, pellets, tablets with immediate and sustained release characteristics, oral fast dissolving systems, transdermal, transmucosal, trans-lingual can be obtained directly as the final dosage form. HME possesses various advantages over other processes as it's a solvent-free technique thus greener and a safer one. Furthermore, since it's a continuous operation, it provides high throughput, few processing steps with online monitoring and minimum processing variables. The products obtained through this process possess better molecular distribution of a drug in the final dosage form thus exhibiting improved bioavailability. The only limitation of HME is that thermolabile compounds cannot be processed due to high processing temperatures [25].

\section{HME- process}

In recent times, HME has been applied in formulation of versatile dosage forms. The process comprises of forceful input of polymers or other raw materials through an orifice under controlled high suitable processing temperature and pressure to yield products with modified physical properties as shown in Fig. 2 [26]. Powder blends comprising of active drugs can be compacted to produce homogenous mixtures in the form of a product of a suitable shape [27]. The flowchart of steps involved in HME of drug and excipients is shown in Fig. 2 [28].

\section{Equipment assembly}

The extrusion equipment commonly used is of three types: Radial screen, ram, roll and screw extruder type. Screw extruders are widely explored in the pharmaceutical industry due to their versatility in

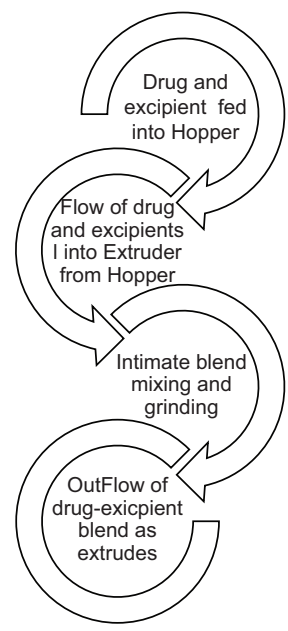

Fig. 2: Flowchart showing steps involved in a hot melt extrusion process yielding products in various forms such as films, rods, and tubes. The extruder equipment mainly comprises of cylindrical barrel stationary in nature and internally classified into different sections where the residence time of the drug-excipient blend to be extruded is enhanced. The end of the barrel can be connected to a die-screw plate which is selected based on extrudate shape. The feed material is forced by screw rotation toward the terminal part of die which forms the site of material softening due to heat production due to friction induction to barrel wall. The feed with high viscosity is forced to connected die as in the last stage of processing [29].

A typical schematic of an extruder design is shown in Fig. 3;

Types of extruder assemblies

Extruders are classified as:

\section{Single-screw extruders (SSEs): Smooth or grooved barrel}

A typical schematic of SSE is shown in Fig. 4 [30,31]. It consists of single screw rotating continuously in a barrel, producing good quality molten material with the consistent output generated by high stable pressure. Screw design generally consists of 20 or more turns with a pitch similar to the screw diameter. Thus, a long slender machine efficient to control longitudinal temperature gradients is created, providing end to end mixing with its considerable residence time. There are three distinct zones: the feed zone, the compression zone and the metering zone.

The compression zone is also known as the processing zone and is accompanied by a few other steps such as mixing, kneading and venting. The raw material fed at once through the feed hopper is received in the feeding area and then conveyed along a flighted screw enclosed in the barrel. The screw rpm in the hopper determines the output rate. If the mass flow rate of the feed system is set independent of the screw rpm, then they may occasionally be fed partly. A flow channel is formed due to rotation of the screw led by the flighting screw and inner surface of the barrel. Frictional forces in the flow channel help the SSE to propel the raw material toward the proximal portion of the screw. Frictional forces in the flow channel help the SSE to propel the raw material toward the proximal portion of the screw. A melt pool is formed as a result of the heated barrel surface and the mechanical energy input supplied by the screws to the raw material. Since the melt pool is equal to the size of the solid bed, it grows as the solid bed shrinks. The molten extrudate is then pumped finally through a die that imparts a definite shape for further downstream processing. The above features along with low cost and low maintenance make it a choice for the production of almost all extruded products.

\section{Twin-screw extruders (TSES)}

Two agitator assemblies are mounted on parallel shafts in TSE. Various configurations can be obtained with two different screws along with different conditions while conveying material in all the zones of the extruder right from the feeding zone through the hopper to the rotating screw and finally to metered pumping zone. Screw arrangement in TSE can be classified as co-rotating (same direction) or counter-rotating (opposite direction), further classifying them as intermeshing or nonintermeshing as depicted in Fig. 5.

The completely intermeshing TSE is a common design with a selfcleaning function that reduces non-motion while also preventing local overheating of raw materials with the extruder. This avoids rotation of raw material along with the screw, preventing its adherence to the firstin/first-out principle of the extruder. The non-intermeshing design is less popular due to its weaker screw interactions leading to improper mixing and also has a lower self-cleaning capability. Its screw arrangement leads to its applicability for highly viscous materials, as they are not susceptible to high torque generation. It can also be used for the removal of large amounts of volatile substances. TSE exhibits the following salient features- reduced residence time, self-cleaning of the screw, minimum supply, enhanced mixing and flexibility in operating functions [29-31]. 


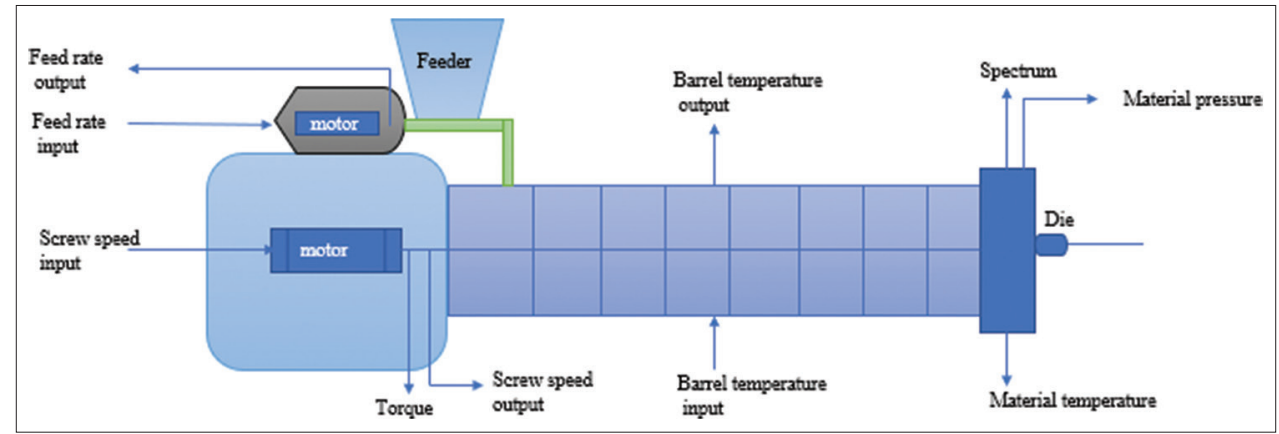

Fig. 3: An extruder design. Adapted from reference [31]

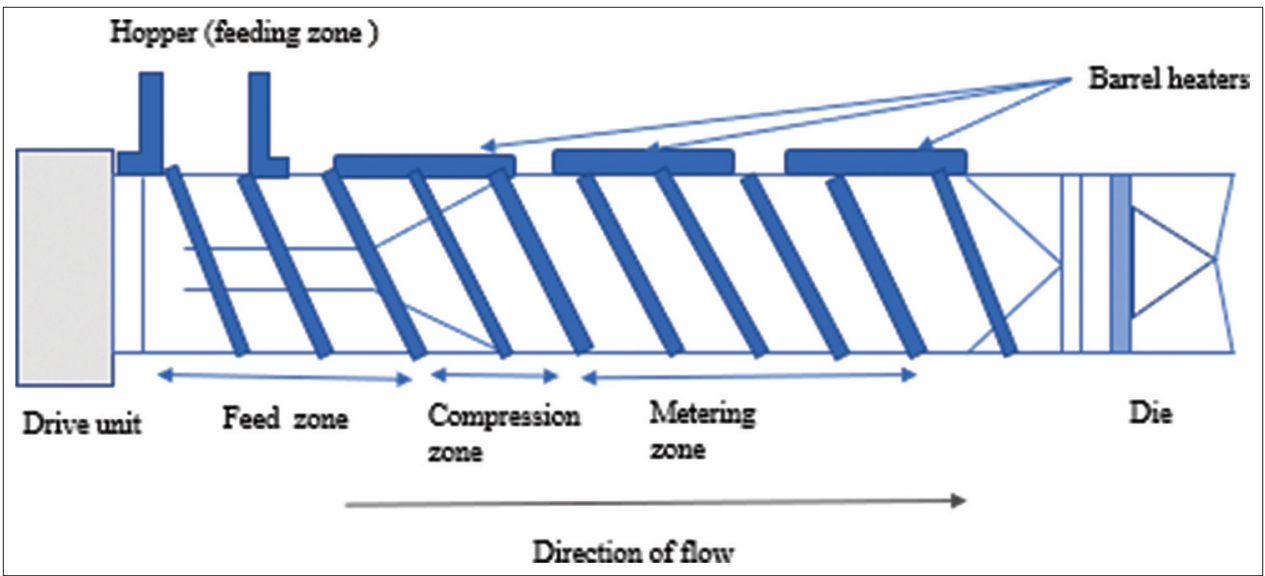

Fig. 4: Single screw extruder. Adapted from reference [30]

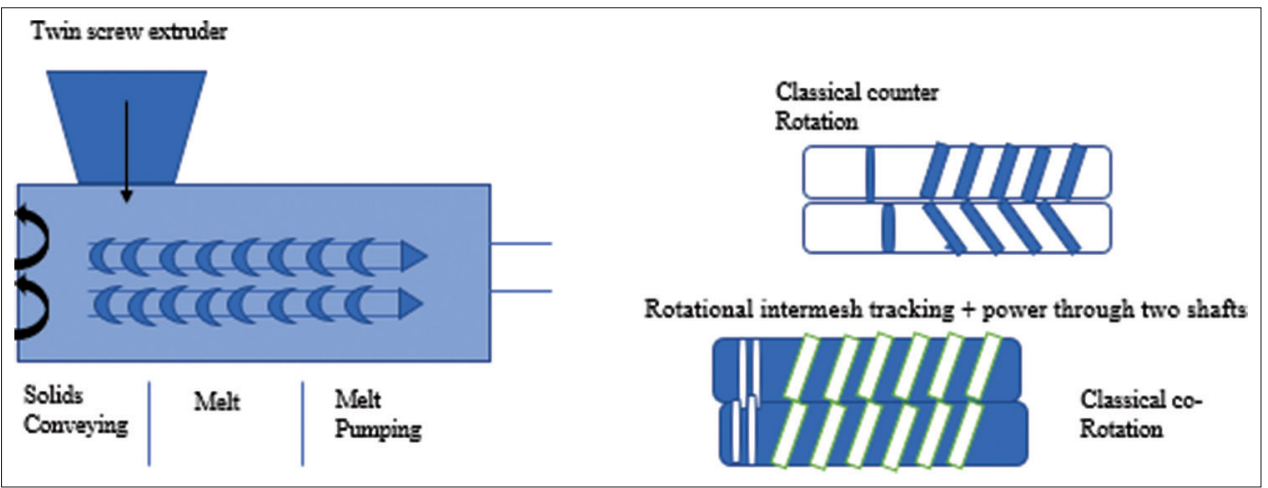

Fig. 5: Twin screw extruder with co- rotating and counter-rotating screws adapted from reference $[31,51,55]$

\section{Multi-screw extruders (MSEs)}

MSE has more than two screws incorporated, and extruder assembly varies depending on the number of these screws. The one with four screws has one control screw and three spurs, while the one with three or five screws is set up linearly. On the other hand, screws in the extruder can be organized circumferentially when they are six to eight in number. The main advantage of MSE over SSE is the prevention of thermal degradation of thermolabile materials, due to its positive displacement flow in the intermeshing region between the screws unlike SSE where the high shear-dominated flow of the melted material is observed.

\section{RAW MATERIALS FOR HME PROCESS}

The salient characteristics observed for hot melt extrudes or hot melt extruded cocrystals are due to the intimate interaction of drug and coformer brought about by the mixing action of screws during HME. Some of the desirable properties for excipients used in HME include: i. Ease of deformation during extrusion and solidification after processing

ii. Thermo stability and maintenance of features during the storage period

iii. Safety and purity when used for drug delivery

iv. Compliance of in vitro release specifications after conversion into dosage forms.

\section{Carriers}

Use of carriers is an indispensable component in HME process as they act as vehicles for drug delivery. Two types of carriers used are polymeric and non-polymeric carriers. As heat processability is an issue for most of the APIs used, polymeric carriers should possess process ability at low temperatures. The drug polymer miscibility plays an important role in carrier selection. The stability of carrier to various processing conditions is also a desirable attribute. The melting characteristics of excipients and polymers used and the binding properties during extrusion process play a key role in imparting desirable characteristics 
to extruded drug-excipient blends. Water-insoluble polymers and waxes such as ethyl cellulose or carnauba wax are commonly used. Desired physicochemical properties of carriers used are as follows:

\section{Thermal properties}

The glass transition temperature and melting temperature of polymers and melting and softening point of excipients are important parameters of consideration during excipient selection for HME. Polymers comprising of repeating units of single monomers or combination of monomers show variable characteristics at the crystalline state. The arrangement at atomic state imparts the polymer with high or low flexibility in chain structure which further affects the extrusion process.

Some thermal properties of polymer affecting HME process include:

a. Thermoplasticity and stability at temperature of extrusion

b. High processing temperature is desirable for polymers and excipients with high $\mathrm{T}_{\mathrm{g}}$ and $\mathrm{T}_{\mathrm{m}}$ which can cause degradation of temperature sensitive molecules

c. Maintenance of processing temperature $20-40^{\circ} \mathrm{C}$ above the glass transition temperature of polymers is adopted during extrusion. However, proper preformulation studies are desirable to predict the interaction profile of drug and excipients

d. Certain auxiliary excipients can be added to facilitate the extrusion process which can act as plasticizers or binding agents [32].

Melt viscosity it is a parameter that aids to identify chain flexibility and degree of entanglement of the polymer used. Several factors affect melt viscosity as depicted in Fig. 6 [32]. Polymers with high melt viscosity drastically an increase the processing torque within the extruder ultimately overloading the motor and the screws. Low melt viscosity leads to a version of the extrudate from the extrusion die. The optimum range of desirable polymer melt viscosity is between 1000 and 10,000 $\mathrm{Pa} \cdot \mathrm{s}[33,34]$

Melt viscosity is also influenced by polymer chain which affects the degree of entanglement and molecular weight of polymer. Higher temperatures lead to chain disentanglement thus reducing the melt viscosity.

\section{Solubilization potential}

Polymer potential to solubilize an active in aqueous solution is known as the solubilization potential of the polymer. Thus, these polymers an increase bioavailability and resorption, to release the API. Some other parameters affecting solubility include drug physicochemical properties, operating conditions, screw configuration, and operating conditions [35].

\section{Mechanical properties}

Mechanical properties of the polymers define the extent to stretch or bent and its hardness which is necessary to optimize the process of HME [36]. Drug product stability and drug release are also affected by the mechanical properties. Factors defining these mechanical properties are:

Strength

- Ultimate elongation (percent elongation to break)

- The young's modulus (modulus of elasticity or tensile modulus).

\section{Viscoelasticity}

Thermoplastic polymers must be used in HME for various dosage forms. Various biodegradable polymers such as polylactic acid, polyglycolic acid, and poly (lactide-co-glycolide) have been used in HME. Along with excipients with low-molecular-weight such as sugars, sugar alcohols and waxes, excipients such as starch and starch derivatives can be used [29].

\section{API}

Crystalline API is preferred over the amorphous for sustained/ controlled-release formulations due to its physical and chemical stability, process ability and availability in diverse forms such as polymorphs, anhydrates, hydrates, and solvates. Drug release varies according to salt form as different crystal lattices impact matrix hydration and permeability. However, to overcome the limitation of poor dissolution which is exhibited by crystalline products, an amorphous form can also be used. However, amorphous form is thermodynamically unstable and thus they are devitrified into crystalline form for storage [29]. Monitoring of $\mathrm{T}_{\mathrm{g}}$ is essential for amorphous products and it is suggested that it should be at least $50^{\circ} \mathrm{C}$ above the storage temperature of the product to maintain stability during its shelf life [37]. API may also alter the functionality of the other components in the formulation along with its thermal degradation. For instance, it was observed that Oxprenolol hydrochloride melted under HME processing conditions and thereby decreased the viscosity of the extrudate, yielding a material with poor handling properties. Lidocaine drug was found to lower the $\mathrm{T}_{\mathrm{g}}$ of Eudragit ${ }^{\circ}$ E/HDPE films $[38,39]$.

\section{Plasticizer}

Plasticizers are low molecular weight compounds that can soften polymers to make them more flexible. It facilitates the HME process to occur at low temperatures preventing the degradation of thermolabile APIs. They modify the extrudate properties during processing which determines the release from the final dosage form. Plasticizers are classified as traditional, non-traditional and special plasticizers.

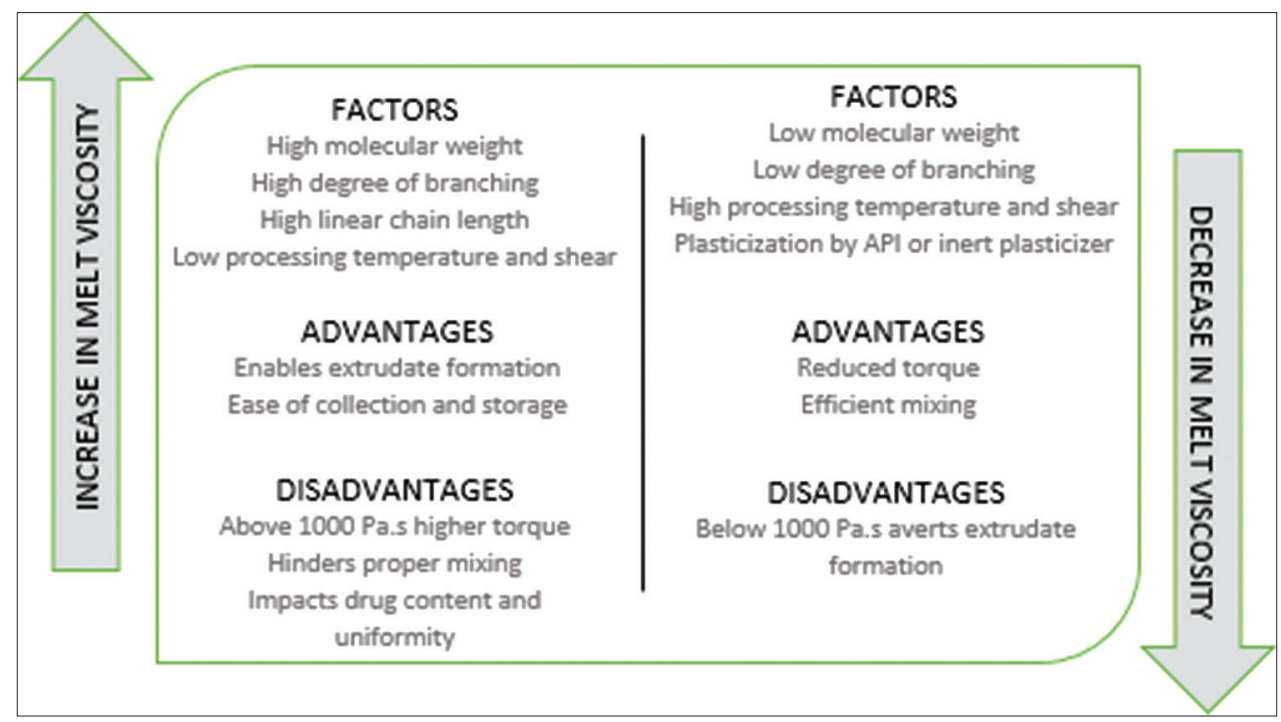

Fig. 6: Factors affecting melt viscosity. Adapted from reference $[24,25]$ 
Traditional plasticizers include Triacetin (most common) citrate ester, Vitamin E D-alpha tocopheryl PEG 1000 succinate (TPGS), surfactants and low molecular-weight polyethylene glycols. Nontraditional plasticizers are added in formulations to serve special purposes [40-43]. Special plasticizers include pressurized $\mathrm{CO}_{2}$ which acts as a foaming agent and also reduces the temperature of various polymers during the process [44-47].

\section{CRITICAL PROCESS PARAMETERS (CPP) IN HME}

\section{Extruder type}

The extruder type influences the overall process efficiency of cocrystallization using the HME technique. For a homogenous blend of drug and coformer and to ensure desirable non-covalent interactions between the two, TSE is an extruder of choice due to the intimate mixing action between the drug and coformer provided by it [48]

\section{Temperature}

The final properties of extrudes are determined by the melt viscosity of the blend in the extruder during processing which is further controlled by temperature of extrusion. General extrusion of drugs and coformers is required to be conducted ensuring that the degradation temperature of the drug and coformer is not exceeded. Hence study on melting point properties of the drug and coformer before the application of HME technique and selection of a suitable extrusion temperature plays an important role in successfully yielding cocrystals.

Extrusion at low temperatures can adversely affect the extruder torque thus affecting the overall mixing capacity. To optimize the process of cocrystallization by extrusion, the melting point of the physical blend of drug and coformer can be determined and the extrusion can be conducted at a temperature higher than the melting temperature of the drug-coformer physical blend [48].

\section{Configuration of screw}

Different screw components of conveying and combining zones change screw structure. The screw diameter (D) and the ratio of screw length (L) to diameter (L/D) are two geometric parameters that define the extruder's design. This ratio plays a major role in optimizing the degree of mixing for obtaining the desired product characteristics. Screw rotation results in the spreading of the feed which ensures its proper dispersive mixing as high shear forces are generated even with lower energy input limiting the degradation of thermolabile materials $[49,50]$. For cocrystal synthesis using HME, minimum of one kneading zone should be present at or near the cylinder end. This aids to generate better quality cocrystals $[51,52]$.

\section{Screw speed}

The speed of screw rotation an increases or decreases the flow rate of the molten mixture inside the barrel modulating its residence time. It is also influenced by the degree of barrel filling and the material's feed rate [53]. High screw speeds can induce amorphization and deterioration of pharmaceutical crystalline materials due to increased friction induced by substantial shear force. From studies, it is found that for good quality cocrystals; low screw rotation speeds should be employed, which will increase the residence time of the material in the extruder.

\section{Feed rate}

Increasing the feed rate of the API coformer mix, keeping the screw rotation speed constant causes more material to be filled in the barrel per unit time. This decreases the material's residence time resulting in increased torque and pressure in the system die's nozzle. All this may block the material in the extruder consequently failing the process. To avoid this, it is essential to optimize the component's feed rate for ensuring the process continuity $[48,49]$.

\section{COCRYSTALLIZATION BY HME}

Medina et al. first introduced the technology of HME for the mechanochemical synthesis of cocrystals. The research concluded that HME possesses advantages such as intense mixing of components with shear leading to plasticization of the material which improves the surface contact between the API and coformer, leading to the synthesis of co-crystals without the presence of a solvent [54].

In HME, API and the coformer are simultaneously combined in a typical molar ratio and are fed to the preheated screw extruder, where melting facilitates the mixing of the starting material. The nucleation of the cocrystals occurs directly in the melt and pure cocrystals are continuously isolated from the extruder. The advantages of the method include the elimination of organic solvents, reduced waste, higher conversion rate, fast operating times compared to solution-based methods and continuous pharmaceutical processing. Indomethacinsaccharin cocrystals were produced by Moradiya et al., employing melt extrusion as a continuous manufacturing technique where it was concluded that the temperature profile, the feeding speed and the screw speed were the three CPP for producing high-quality cocrystals [55]. It was also demonstrated that temperature has no impact on the dissolution rate of cocrystals. However, according to Rietveld's analysis, it can be valid only for samples that have the same crystallinity and the dissolution can be affected if there is a deviation in crystal quality due to the process temperature. The dissolution profile can also be affected by the particle size. Dhumal et al. manufactured ibuprofen-nicotinamide agglomerated cocrystal by HME. The study concluded that for cocrystal formation, the temperature of the barrel must be above the eutectic point of the physical mixture and the highest shear screw configuration must be applied to obtain purer cocrystals $[56,57]$.

\section{Synthesis of cocrystals using primary components, that is, API and a coformer}

Identifying and optimizing the CPPs in HME assisted cocrystal synthesis

Medina et al. synthesized anhydrous caffeine: oxalic acid dihydrate cocrystals (2:1) using HME. Extrusion was carried out using a corotary TSE mounted in extruder dies with a screw diameter of $16 \mathrm{~mm}$ (25:1 L/D) but without a nozzle. Parameters such as temperature, screw configuration and residence time were monitored for evaluation and optimization of the conditions required to synthesize cocrystals. The melting temperatures of caffeine is $236.28^{\circ} \mathrm{C}$ and oxalic acid is $99.28^{\circ} \mathrm{C}$ and thus extruder temperature was maintained at $75^{\circ} \mathrm{C}$ throughout the screw zones which was lower than the individual components at a 5\% volumetric mix feed rate and the screw speed was set to $100 \mathrm{rpm}$.

The cocrystals synthesized exhibited a superior performance which was confirmed by near-infrared spectroscopy (NIR) [58]. Furthermore, the cocrystals produced by this method had better bulk density and flow characteristics than those obtained by solution cocrystallization. Ibuprofen: nicotinamide cocrystal was synthesized by Dhumal et al., using same extruder of screw diameter $16 \mathrm{~mm}(40: 1 \mathrm{~L} / \mathrm{D})$ with preset barrel temperatures, screw speeds $(20,30$, and $40 \mathrm{rpm})$ and screw configurations. Melting point of the eutectic mixture of drug: coformer (1:1) was found to be $74^{\circ} \mathrm{C}$. As a result, the physical mixtures were extruded with three separate screw configurations both above $80^{\circ} \mathrm{C}$ and $90^{\circ} \mathrm{C}$ and below $70^{\circ} \mathrm{C}$; the eutectic melting point. A-with conveyer to ensure minimal mixing pressure, B-with mixing areas, and $\mathrm{C}$-with dispersion blend, allowing for high cutting powers, to evaluate the influence of temperature, mixing and cutting speed on the efficiency of the extrusion process for the mechanochemical synthesis of cocrystals $[54,59]$.

PXRD diffractograms of mixtures processed at $70^{\circ} \mathrm{C}$ for these three configurations indicated inadequate conversion of the reaction mixture to the cocrystal with a high proportion of unreacted ibuprofen, while those processed at $80^{\circ} \mathrm{C}$ and $90^{\circ} \mathrm{C}$ revealed a comparatively high percentage of extruded cocrystal. This demonstrates that molten mixtures allow cocrystallization due to accelerated mixing and mass transfer, resulting in nucleation and further growth of cocrystal nuclei during molten mixture solidification. The effect of screw speed was evaluated on extrusion conducted at $80^{\circ} \mathrm{C}$ and $90^{\circ} \mathrm{C}$. The lower screw speed allowed the material to stay in the extruder for a longer period 
increasing the yield of the cocrystals at both temperatures, resulting in maximum yield and improved output for batches processed at $90^{\circ} \mathrm{C}$ as calculated by PXRD research. As compared to configuration A, configuration $\mathrm{B}$ demonstrated an improvement in mixing rate at both temperatures, resulting in a higher yield of the obtained cocrystal.

While the C-screw configuration produced cocrystals of the highest purity as verified by PXRD review. This design with a screw speed of $20 \mathrm{rpm}$ at $90^{\circ} \mathrm{C}$ demonstrated the full API transformation into cocrystal verifying the effect of both the process temperature and the low screw speed on the final product's phase purity. Hence the above study concluded that an increase in temperature decrease the viscosity of molten material resulting in greater mass transfer and better interaction thus, enhancing the performance of cocrystal. The different screw configurations aid in enhanced mixing and increasing the cutting speed to improve the efficiency of cocrystallization. Studies by Paradkar et al. and group showed that repeated exposure of reacting components to high pressure and shear stress was the most significant factor in cocrystal production. There is a need to optimize the time required for this process to avoid API degradation. It is critical that the mixing regions account for at least $1 / 40$ of the overall screw duration and the screw contains at least one distributive blending unit followed by at least one dispersive mixing element. Distributive mixing regions must be at least $1 \mathrm{~L} / \mathrm{D}$ in length, along with each dispersive mixing region having at least $0.5 \mathrm{~L} / \mathrm{D}[60]$.

\section{Phase control in cocrystal synthesis through HME}

Stoichiometric control of cocrystal synthesis was performed by Kulkarni et al. Cocrystals of caffeine: maleic acid was synthesized using HME in the ratio $1: 1$ and $2: 1$ to achieve phase control by avoiding interconversion between both ratios of caffeine: maleic acid. The process was carried out using TSE having a screw diameter of $16 \mathrm{~mm}$, L/D ratio 40:1, and screw speed $10 \mathrm{rpm}$. This screw configuration generated intense cutting speed and a long residence time of material blend in an extruder. Caffeine: maleic acid $1: 1$ blend was processed at $80^{\circ} \mathrm{C}, 90^{\circ} \mathrm{C}$ and $100^{\circ} \mathrm{C}$, whereas the $2: 1$ mixture was processed at $100^{\circ} \mathrm{C}, 105^{\circ} \mathrm{C}$, and $110^{\circ} \mathrm{C}$. These temperatures mimicked the DSC endothermic events for eutectic formation for both cocrystals and were accessed below and above the eutectic points. The process demonstrated that a $1: 1$ ratio yielded a pure cocrystal phase at $100^{\circ} \mathrm{C}$ which is the eutectic point of the physical mixture. At the same time, pure cocrystal phase for $2: 1$ ratio was obtained at $110^{\circ} \mathrm{C}$ only as melting temperature of the $1: 1$ cocrystal is $104^{\circ} \mathrm{C}$ and below that temperature an intermediate is formed after which melted material can react freely to form stable cocrystal. Thus, this study stated the importance of extrusion parameters, that is, the temperature for controlling the stoichiometry of synthesized crystals [61].

\section{Suspension of cocrystal in an inert matrix}

The addition of API and a coformer in an inert matrix facilitates the reaction during cocrystal synthesis using HME. The inert matrix is selected based on the difference between the Hansen Solubility Parameter values $(\triangle \mathrm{HSP})$ of cocrystals and the inert matrix. In general, for cocrystallization to occur the difference in HSP between API and coformer should be less than $7 \mathrm{MPa} 0.5$, however for cocrystal formation in an inert matrix; is should be greater than 9.6 MPa0.5 allowing an increase in the content of inert matrix in the formulation [62]. Thus, this is used as a predictive tool for the formation of cocrystals in the formulation design stage. This neutral matrix is just a functional vehicle for formulating matrix mixtures using one step HME process which does not evaporate, but only solidifies after cooling. The primary requirement for obtaining good quality cocrystal is that the cocrystal components should have significantly reduced miscibility/solubility inside the inert carrier $[63,58]$. There exist several principal matrix parameters influencing successful synthesis and formulation of pharmaceutical cocrystals as given in Fig. 7.

Observations about extrusion torque and residence time were deduced by Li et al. Ibuprofen: isonicotinamide cocrystals were synthesized using HME with xylitol using different concentrations (10\%, 30\%, and 50\%) [64]. Xylitol being low molecular weight facilitates cocrystallization due to its limited miscibility with the reaction mixture. Furthermore, it has a low melting point of $92^{\circ} \mathrm{C}$ as well as low melting viscosity which makes it favorable for HME. It was hypothesized that high-quality cocrystals could be obtained only when the matrix remain separated from individual cocrystal components not disturbing the formation of non-covalent bonds between API and coformer. Hence, the extrusion process was carried out in two stages for avoiding early phase separation and possible blocking of the extruder while emptying the barrel. The feeding and mixing stage was performed at $92^{\circ} \mathrm{C}$ with the screw rotating at $10 \mathrm{rpm}$. During the washing stage, the temperature was reduced by $7^{\circ} \mathrm{C}$ and the screw speed was increased to $50 \mathrm{rpm}$.

Both torque and the residence time of the mixture in the cylinder was found to change, depending on the weight fraction of xylitol in the formulation. After addition of $10 \% \mathrm{w} / \mathrm{w}$ of xylitol, torque was increased from 40-44 $\mathrm{Ncm}$ to $109-122 \mathrm{Ncm}$ with a reduction in residence time from $233 \mathrm{sec}$ to $90 \mathrm{sec}$ on comparing with processing of pure crystal components. This increased the efficiency of cocrystallization from $28.1 \%$ to $33.5 \%$ which was confirmed by PXRD analysis. Further increasing xylitol concentration to $30 \%$ and $50 \% \mathrm{w} / \mathrm{w}$ the reduction in torque was obtained to $43-59 \mathrm{Ncm}$ and 19-22 Ncm respectively along with an increase in residence time to $272 \mathrm{~s}$ and $329 \mathrm{~s}$. However, at these concentrations, the efficiency of the process did not change resulting in a conversion of $28.6 \%$ and $28.3 \%$ of the mixture to the cocrystal respectively. Thus, highest efficiency was attributed to lack of miscibility between the crystal and xylitol and low viscosity of the melt mix in the extruder providing greater mobility to the molecules to react in the extrudate mixture. The inert molten carrier functions are similar to solvent in LAG with the only difference being inability to remove the carrier after extrusion due to its non-volatile nature. The low melt viscosity of xylitol and its rapid solidification on extrusion leads to the uniform distribution of the cocrystal in the matrix. Furthermore, cocrystals obtained using this method showed an improved dissolution rate with an increased weight fraction of xylitol in the final formulation [64].

Further optimization of process parameters, that is, the temperature and screw configuration was also done by Li et al. To study miscibility in the matrix, the difference in theoretical values of Hansen solubility parameters was studied where calculated theoretical values confirmed excellent miscibility of ibuprofen with iso-nicotinamide $(\Delta \delta \mathrm{t} 1.95 \mathrm{MPa} 1 / 2)$. The calculated $\Delta \delta \mathrm{t}$ value of $13.74 \mathrm{MPa} 1 / 2$ for the cocrystal/xylitol system, guarantee free precipitation of cocrystal in the matrix on extrudate solidification. This is because high miscibility ensures contact between cocrystal components, ensuring cocrystallization. Hence it is crucial to identify proper mixing zone with high temperatures for process efficiency. Binary phase diagrams were also studied to access the miscibility of components [63]. The analysis indicated that the miscibility of ibuprofen and xylitol decreases with an increase in temperature above $95^{\circ} \mathrm{C}$. A similar relationship was observed for the ibuprofen-isonicotinamide mixture above $115^{\circ} \mathrm{C}$. However, for isonicotinamide xylitol system, the components miscibility increased above $110^{\circ} \mathrm{C}$. Thus, this miscibility analysis proved to be important in determining the exact temperature for extrusion [48].

\section{Cocrystals incorporated in a polymer matrix}

This method is similar to previous techniques. Cocrystal synthesis takes place in the presence of an inert excipient to improve the physical and chemical stability of the product. This method is called polymer-assisted grinding (POLAG). Polymers are used as catalysts for cocrystallization reaction. They are used in quantity up to $15 \% \mathrm{w} / \mathrm{w}$ $[22,65]$. This method eliminates the risk of formation of solvates which is undesirable and also helps to achieve control over the dissolution rate of the final formulation. When the drug coformer mixture is processed in a solid matrix, the temperature range of the extruder is pre-set to enable melting of matrix and cocrystallization occurs in that melt [66].

Different polymers are used for this purpose. The relation between the cocrystal components and the polymer must be determined. The 


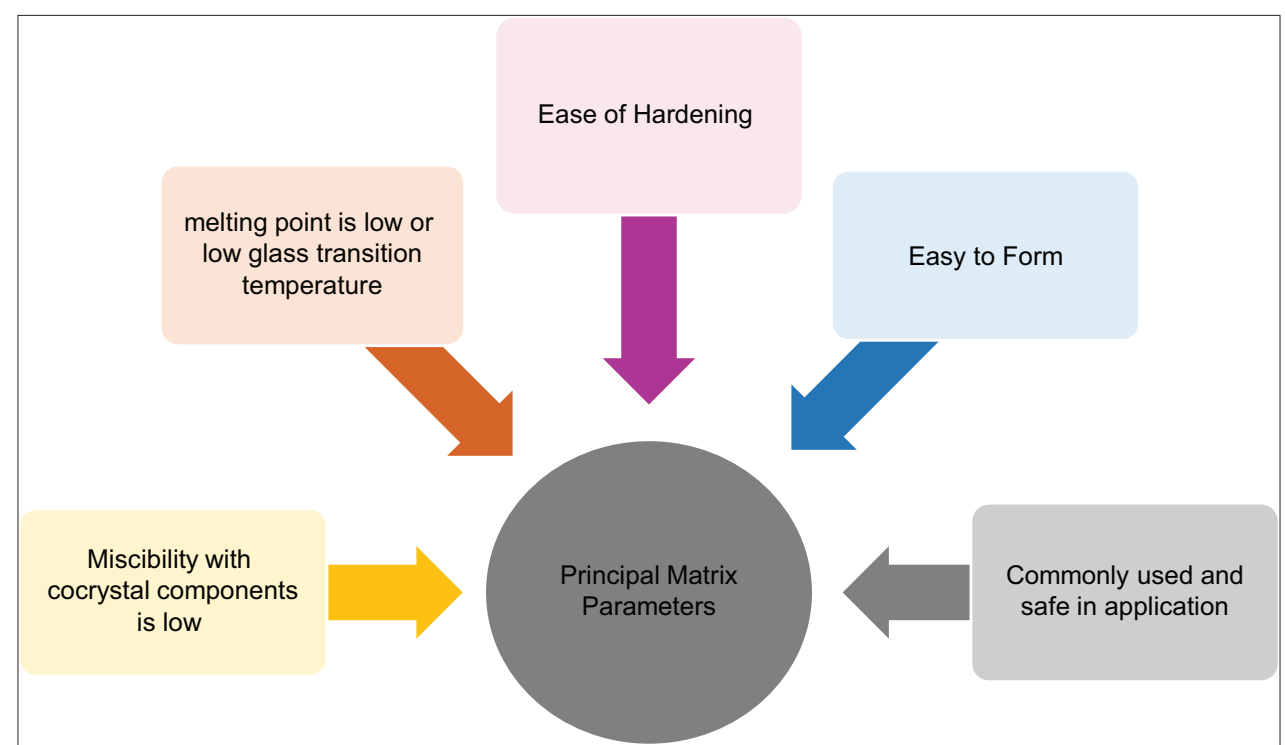

Fig. 7: Principal matrix parameters for formulation of pharmaceutical cocrystals. Adapted from reference [48]

polymer selection should be done depending on its physicochemical properties, that is, $\mathrm{T}_{\mathrm{m}}$ or $\mathrm{T}_{\mathrm{g}}$, and the presence of functional groups capable of forming hydrogen bonds with other cocrystal components and mutual miscibility and solubility of the mixture components [67]. Types of polymers used include amorphous polymers and semicrystalline polymers. Amorphous polymers are used in matrixassisted cocrystallization (MAC). Examples of these polymers include; Soluplus, Eudragit EPO, HPMC and PVP while that of semicrystalline polymers include Poloxamer P407, PEG, and PVA 48.

\section{PROCESS ANALYTICAL TECHNOLOGY (PAT) IN MONITORING OF COCRYSTAL SYNTHESIS}

Application of HME process in cocrystallization can further be advanced using Quality by design (QbD) approach. Application of QbD imparts a systematic stratified approach to generation of cocrystals with desirable properties. Use of knowledge on known parameters to evaluate the parameter effect and determine the design space for a robust cocrystal formulation is one of the classic advantages of cocrystal. The knowledge of different interaction factors helps to overcome the fallacies observed for one factor at a time approach of formulation designing.

Obtaining continuous feedback during the manufacturing process can reduce the risk of the product being rejected due to unspecified results. By implementation of PAT approaches as an important component of the QbD concept, HME process can be easily transferred from a laboratory to a commercial scale; increasing its value in novel pharmaceutical drug supply systems [68].

Use of in process quality control techniques such as Ultraviolet spectroscopy, NIR, X-ray diffraction, and Raman spectroscopy aids to give detailed information on the crystalline drug parameters attempted to modify using cocrystallization approach [69]. Use of PAT aids to alleviate the high chances of batch rejection and failure of crystallized cocrystal formulations by providing appropriate information during the process of formulation designing [48-51].

\section{CONCLUSION}

The pharmaceutical industry generally faces a lot of challenges in pharmaceutical processing of drugs having low solubility issues. This leads to increased investment in R and D and clinical trials and also increases the time required for developing new drugs. It has been observed that various existing pharmaceutical processes are non-continuous involving several batches with a lot of steps increasing the complexity of the process. Cocrystal synthesis of poorly soluble actives can help to overcome solubility and stability issues. However, most of the techniques used for cocrystal synthesis use solvents raising toxicity issues.

Due to these underlying issues of solvent-based cocrystal synthesis, recently HME has emerged as the most desirable technique for cocrystal synthesis as it involves continuous processing and eliminates the need for a solvent. The use of sophisticated instruments such as Twin Screw Extruder contributes to fast processing. The above features make the process scalable and reduce the cost of production. Cocrystals produced through this process show better performance. Further by applying PAT $\mathrm{QbD}$ analysis, the product properties can be monitored online. Due to growing formulation demands, a need has arised to develop advanced characterization techniques which aid to determine and monitor torque values, changes in temperature and interaction profile of drug with excipients. Furthermore, the introduction of POLAG has generated a new era as it exhibits flexibility to use a variety of amorphous and semicrystalline polymers which has enhanced the process effectivity.

Thus, HME has emerged out to be one of the advanced solvent-free techniques for easy processing of drugs to form cocrystals with enhanced performance.

\section{ACKNOWLEDGMENT}

The authors are thankful to Bombay College of Pharmacy where the theme and draft of manuscript was conceptualized, prepared and finalized.

\section{AUTHORS CONTRIBUTION}

The theme of the manuscript was conceptualized by Dr. Ujwala Shinde. The preliminary draft of the manuscript was framed by Ms. Archana Rajadhyax. The finalization of the manuscript draft, formatting and editing of the manuscript was done by Dr. Harita Desai and Ms. Shrushti Mane.

\section{CONFLICTS OF INTERESTS}

The authors declare no conflicts of interest.

\section{AUTHORS FUNDING}

Nil.

\section{REFERENCES}

1. Kawabata Y, Wada K, Nakatani M, Yamada S, Onoue S. Formulation design for poorly water-soluble drugs based on biopharmaceutics 
classification system: Basic approaches and practical applications. Int J Pharm 2011;420:1-10.

2. Haltner E, Flototto T, Bock U. Biopharmaceutical Classification System. Farm Vestn 2003;54:317-18.

3. Kadam SV, Shinkar DM. Review on solubility enhancement technique. Int J Pharm BiolSci 2013;3:462-75.

4. Savjani KT, Gajjar AK, Savjani JK. Drug solubility: Importance and enhancementtechniques. ISRN Pharm 2012;2012:195727.

5. Loftsson T, Brewster ME. Pharmaceutical applications of cyclodextrins. 1. Drug solubilization and stabilization. J Pharm Sci 1996;85:1017-25.

6. Rodríguez-Spong B, Price CP, Jayasankar A, Matzger AJ, RodríguezHornedo N. General principles of pharmaceutical solid polymorphism: A supramolecular perspective Adv Drug Deliv Rev 2004;56:241-74.

7. Rasenack N, Muller BW. Micron-Size drug particles: Common and novel micronization techniques. Pharm Dev Technol 2004;9:1-13.

8. Yu L. Amorphous pharmaceutical solids: Preparation, characterization and stabilization. Adv Drug Deliv Rev 2001;48:27-42.

9. Subramanian S, Zaworotko MJ. Manifestations of noncovalent bonding in the solid state. 6 . [H 4 (Cyclam)] $4+$ (Cyclam $=$ 1,4,8,11-Tetraazacyclotetradecane) as a template for crystal engineering of network hydrogen-bonded solids. Can J Chem 1995;73:414-24.

10. Parkin IP. Supramolecular Chemistry. In: Applied Organometallic Chemistry. Hoboken, New Jersey: John Wiley and Sons Ltd.; 2001. p. 236 .

11. Blagden N, de Matas M, Gavan PT, York P. Crystal engineering of active pharmaceutical ingredients to improve solubility and dissolution rates. Adv Drug Deliv Rev 2007;59:617-30.

12. Desiraju GR. Supramolecular synthons in crystal engineering a new organic synthesis. Angew Chem 1995;34:2311-27.

13. Fukte SR, Wagh MP, Rawat S. Coformer selection: An important tool in cocrystal formation. Int J Pharm Pharm Sci 2014;6:9-14.

14. Dirksen JA, Ring TA. Fundamentals of crystallization: Kinetic effects on particle size distributions and morphology. Chem Eng Sci 1991;46:2389-427.

15. Adhiyaman R, Basu SK. Crystal modification of dipyridamole using different solvents and crystallization conditions. Int $\mathrm{J}$ Pharm 2006;321:27-34

16. Nokhodchi A, Bolourtchian N, Dinarvand R. Crystal modification of phenytoin using different solvents and crystallization conditions. Int $\mathrm{J}$ Pharm 2003;250:85-97.

17. Paradkar A, Maheshwari M, Kamble R, Grimsey I, York P. Design and evaluation of celecoxib porous particles using melt sonocrystallization. Pharm Res 2006;23:1395-400

18. Manish M, Harshal J, Anant P. Melt sonocrystallization of ibuprofen: Effect on crystal properties. Eur J Pharm Sci 2005;25:41-8.

19. Hu J, Johnston KP, Williams RO. Nanoparticle engineering processes for enhancing the dissolution rates of poorly water soluble drugs. Drug Dev Ind Pharm 2004;30:233-45.

20. Trask AV, Motherwell WD, Jones W. Solvent-Drop grinding: Green polymorph control of cocrystallisation. Chem Commun 2004;4:890-91.

21. Karagianni A, Malamatari M, Kachrimanis K. Pharmaceutical cocrystals: New solid phase modification approaches for the formulation of Apis. Pharmaceutics 2018;10:1-30.

22. Douroumis D, Ross SA, Nokhodchi A. Advanced methodologies for cocrystal synthesis. Adv Drug Deliv Rev 2017;117:178-95.

23. Sopyan I, Fudholi A, Muchtaridi M, Puspitasari I. A simple effort to enhance solubility and dissolution rate of simvastatin using cocrystallization. Int J Pharm Pharm Sci 2016;8:342-6.

24. Repka MA, Shah S, Lu J, Maddineni S, Morott J, Patwardhan K, et al. Melt extrusion: Process to product. Exp Opin Drug Deliv 2012;9:105-25.

25. Repka MA, Langley N, Dinunzio JC. Melt extrusion: Materials, technology and drug product design. In: Repka MA, Langley N, JD, editors. AAPS Advances in the Pharmaceutical Sciences Series. $9^{\text {th }}$ ed. New York: Springer; 2013. p. 3-46.

26. Maniruzzaman M, Boateng JS, Snowden MJ, Douroumis D. A review of hot-melt extrusion: Process technology to pharmaceutical products. ISRN Pharm 2012;2012:436763.

27. Breitenbach J. Melt Extrusion: From process to drug delivery technology. Eur J Pharm Biopharm 2002;54:107-17.

28. Chokshi HZ. Hot-Melt extrusion technique: A review. Iran J Pharm Res 2004:3:3-16.

29. Patil H, Tiwari RV, Repka MA. Hot-melt extrusion: From theory to application in pharmaceutical formulation. AAPS PharmSciTech 2016;17:20-42.

30. Andrews GP, Margetson DN, Jones DS, McAllister MS, Diak OA. HotMelt Extrusion: an emerging drug delivery technology. Pharm Technol Eur 2009;21:24-7.
31. Twin-Screw WT. Extrusion and screw design. In: Ghebre-Sellassie I, Martin CE, Zhang F, Dinunzio J, editor. Pharmaceutical Extrusion Technology. New York: Marcel Dekker; 2003. p. 69-98.

32. Thakkar R, Pillai A, Ashour EA, Repka MA. Systematic screening of pharmaceutical polymers for hot melt extrusion processing: A Comprehensive review. Int J Pharm 2020;576:1-45.

33. Kolter K, Karl M, Gryczke A. Physico-chemical characteristics processability. In: Hot-melt Extrusion with Basf Pharma Polymers. Germany: BASF the Chemical Company; 2012. p. 103-23.

34. Gilbert M. Relation of structure to thermal and mechanical properties. In: Brydson's Plastics Materials. $8^{\text {th }}$ ed. Amsterdam, Netherlands: Elsevier; 2016. p. 59-73.

35. Shah S, Maddineni S, Lu J, Repka MA. Melt Extrusion with poorly soluble drugs. Int J Pharm 2013;453:233-52.

36. Balani K, Verma V, Agarwal A, Narayan R. Physical, thermal, and mechanical properties of polymers. In: Balani K, Verma V, Agarwal A, editors. Biosurfaces: A Materials Science and Engineering Perspective. Hoboken, New Jersey: John Wiley and Sons, Inc.; 2015. p. 329-44.

37. Hancock BC, Zografi G. Characteristics and significance of the amorphous state in pharmaceutical systems. J Pharm Sci 1997;86:1-12.

38. Follonier N, Doelker E, Cole ET. Evaluation of Hot-Melt extrusion as a new technique for the production of polymer-based pellets for sustained release capsules containing high loadings of freely soluble drugs. Drug Dev Ind Pharm 1994;20:1323-339.

39. Aitken-Nichol C, Zhang F, McGinity JW. Hot Melt Extrusion of acrylic films. Pharm Res 1996;13:804-8.

40. De Brabander C, Van Den Mooter G, Vervaet C, Remon JP. Characterization of Ibuprofen as a nontraditional plasticizer of ethyl cellulose. J Pharm Sci 2002;91:1678-85.

41. Six K, Berghmans H, Leuner C, Dressman J, Van Werde K, Mullens J, et al. Characterization of solid dispersions of itraconazole and hydroxypropylmethylcellulose prepared by melt extrusion, Part II. Pharm Res 2003;20:1047-54.

42. Verreck G, Six K, Van den Mooter G, Baert L, Peeters J, Brewster ME. Characterization of solid dispersions of itraconazole and hydroxypropylmethylcellulose prepared by melt extrusion Part I. Int J Pharm 2003;251:65-74.

43. Lakshman JP, Cao Y, Kowalski J, Serajuddin AT. Application of Melt extrusion in the development of a physically and chemically stable high-energy amorphous solid dispersion of a poorly water-soluble drug. Mol Pharm 2008;5:994-1002.

44. Kazarian SG. Polymer processing with supercritical fluids. Polym Sci Ser C 2000;42:78-101

45. Kiran E. Polymer formation, modifications and processing in or with supercritical fluids. In: Kiran E, editor. Supercritical Fluids. Dordrecht: Springer; 1994. p. 541-8

46. Chiou JS, Barlow JW, Paul DR. Plasticization of glassy polymers by $\mathrm{CO}_{2}$. J Appl Polym Sci 1985;30:2633-42.

47. Verreck G, Decorte A, Li H, Tomasko D, Arien A, Peeters J, et al. The effect of pressurized carbon dioxide as a plasticizer and foaming agent on the hot melt extrusion process and extrudate properties of pharmaceutical polymers. J Supercrit Fluids 2006;38:383-91.

48. Gajda M, Nartowski KP, Pluta J, Karolewicz B. Continuous, one-step synthesis of pharmaceutical cocrystals via Hot melt extrusion from neat to matrix-assisted processing state of the art. Int J Pharm 2019;558:426-40.

49. Thiry J, Krier F, Evrard B. A review of pharmaceutical extrusion: Critical process parameters and scaling-up. Int J Pharm 2015;479:227-40.

50. Martin C. Twin Screw extruders as continuous mixers for thermal processing: A technical and historical perspective. AAPS PharmSciTech 2016;17:3-19.

51. Moradiya HG, Islam MT, Halsey S, Maniruzzaman M, Chowdhry BZ, Snowden MJ, et al. Continuous cocrystallisation of carbamazepine and trans-cinnamic acid via melt extrusion processing. Cryst Eng Commun 2014;16:3573-83.

52. Paradkar A, Dhumal RS, Kelly AL, York P, Coates PD. Cocrystallization and simultaneous agglomeration using hot melt extrusion. Pharm Res 2010;27:2725-33

53. Reitz E, Podhaisky H, Ely D, Thommes M. Residence time modeling of hot melt extrusion processes. Eur J Pharm Biopharm 2013;85:1200-5.

54. Medina C, Daurio D, Nagapudi K, Alvarez-Nunez F. Manufacture of pharmaceutical cocrystals using twin screw extrusion: A solvent-less and scalable process. J Pharm Sci 2010;99:1693-6.

55. Moradiya H, Islam MT, Woollam GR, Slipper IJ, Halsey S, Snowden MJ, et al. Continuous cocrystallization for dissolution rate optimization of a poorly water soluble drug Cryst Growth Des 2014;14:189-98.

56. Moradiya HG, Islam MT, Scoutaris N, Halsey SA, Chowdhry BZ, Douroumis D. Continuous manufacturing of high quality pharmaceutical 
cocrystals integrated with process analytical tools for in-line process control. Cryst Growth Des 2016;16:3425-34.

57. Karimi-Jafari M, Padrela L, Walker GM, Croker DM. Creating cocrystals: A review of pharmaceutical cocrystal preparation routes and applications. Cryst Growth Des 2018;18:6370-87.

58. Walsh D, Serrano DR, Worku ZA, Madi AM, O'Connell P, Twamley B, et al. Engineering of pharmaceutical cocrystals in an excipient matrix: Spray drying versus hot melt extrusion. Int J Pharm 2018;551:241-56.

59. Bak A, Gore A, Yanez E, Stanton M, Tufekcic S, Syed R, et al. The cocrystal approach to improve the exposure of a water-insoluble compound: AMC 517 sorbic acid cocrystal characterization and pharmacokinetics. J Pharm Sci 2008;97:3942-56.

60. Anant P, Adrian K, Phil C, Peter Y. Method and Product WO 2010/013035 A1; 2010.

61. Kulkarni C, Wood C, Kelly AL, Gough T, Blagden N, Paradkar A. Stoichiometric control of cocrystal formation by solvent free continuous cocrystallization (sfcc). Cryst Growth Des 2015;15:5648-51.

62. Mohammad MA, Alhalaweh A, Velaga S ZP. Hansen solubility parameter as a tool to predict cocrystal formation. Int J Pharm 2011;407:63-71.

63. Li S, Yu T, Tian Y, Lagan C, Jones DS, Andrews GP. Mechanochemical synthesis of pharmaceutical cocrystal suspensions via Hot melt extrusion: Enhancing cocrystal yield. Mol Pharm 2018;15:3741-54.

64. Li S, Yu T, Tian Y, McCoy CP, Jones DS, Andrews GP. Mechanochemical synthesis of pharmaceutical cocrystal suspensions via Hot melt extrusion: Feasibility studies and physicochemical characterization. Mol Pharm 2016;13:3054-68.

65. Hasa D, Carlino E, Jones W. Polymer-assisted grinding, a versatile method for polymorph control of cocrystallization. Cryst Growth Des 2016;16:1772-9.

66. Boksa K, Otte A, Pinal R. Matrix-assisted cocrystallization (MAC) simultaneous production and formulation of pharmaceutical cocrystals by hot-melt extrusion. J Pharm Sci 2014;103:2904-10.

67. Gajda M, Nartowski KP, Pluta J, Karolewicz B. The role of the polymer matrix in solvent-free hot melt extrusion continuous process for mechanochemical synthesis of pharmaceutical cocrystal. Eur J Pharm Biopharm 2018;131:48-59.

68. Hitzer P, Bauerle T, Drieschner T, Ostertag E, Paulsen K, van Lishaut H, et al. Process Analytical techniques for hot-melt extrusion and their application to amorphous solid dispersions. Anal Bioanal Chem 2017;409:4321-33

69. Wesholowski J, Prill S, Berghaus A, Thommes M. Inline UV/Vis spectroscopy as PAT tool for hot-melt extrusion. Drug Deliv Transl Res 2018;8:1595-603. 